\title{
Perfect Simulation and Stationarity of a Class of Mobility Models
}

\author{
Jean-Yves Le Boudec and Milan Vojnović
}

\begin{abstract}
We define "random trip", a generic mobility model for independent mobiles that contains as special cases: the random waypoint on convex or non convex domains, random walk with reflection or wrapping, city section, space graph and other models. We use Palm calculus to study the model and give a necessary and sufficient condition for a stationary regime to exist. When this condition is satisfied, we compute the stationary regime and give an algorithm to start a simulation in steady state (perfect simulation). The algorithm does not require the knowledge of geometric constants. For the special case of random waypoint, we provide for the first time a proof and a sufficient and necessary condition of the existence of a stationary regime. Further, we extend its applicability to a broad class of non convex and multi-site examples, and provide a ready-to-use algorithm for perfect simulation. For the special case of random walks with reflection or wrapping, we show that, in the stationary regime, the mobile location is uniformly distributed and is independent of the speed vector, and that there is no speed decay. Our framework provides a rich set of well understood models that can be used to simulate mobile networks with independent node movements. Our perfect sampling is implemented to use with ns-2, and it is freely available to download from http://ica1www.epfl.ch/RandomTrip.
\end{abstract}

\section{INTRODUCTION}

\section{A. Mobility Models and Stationarity}

Our goal is to provide a class of mobility models (1) that is rich enough to accommodate a large variety of examples and (2) whose simulation can easily be mastered. The latter point is motivated by recent findings about the random waypoint, an apparently simple model that fits in our framework. The simulation of the random waypoint poses a surprising number of challenges, such as speed decay, a change in the distribution of location and speed as the simulation progresses [16], [12], [14], [8]. All of these observations are related to the existence of a stationary regime. Camp, Navidi and Bauer [14] point out that if the model has a stationary regime, it is

Author affiliations: Jean-Yves Le Boudec, EPFL, CH-1015, Lausanne, Switzerland, Email: jean-yves.leboudec@epfl.ch; Milan Vojnović, Microsoft Research Ltd, CB3 OFB Cambridge, United Kingdom, Email: milanv@microsoft.com. important to simulate it in this regime; otherwise, if the initial configuration is not sampled from the stationary regime, the performance evaluation of a system under study may be biased and non reproducible.

\section{B. Perfect Simulation}

A standard method for avoiding such a bias is to (1) make sure the used model has a stationary regime and (2) remove the beginning of all simulation runs in the hope that long runs converge to stationary regime. However, as we show now, the length of transients may be prohibitively long for even simple mobility models. Our example is the space graph explained in Figure 1. There are a little less than 5000 possible paths; in Figure 1 we show the distribution of the path used by the mobile at time $t$, given that initially a path is selected uniformly among all possible paths (i.e. the mobile is initially placed uniformly among all nodes). This was obtained analytically (see Appendix [4] for details). Figure 1 illustrates that the transient period may be long compared to typical simulation lengths (for example $900 \mathrm{sec}$ in [5]). A major difficulty with transient removal is to know when the transient ends; if it may be long, as we illustrated, considerable care should be used. An alternative, called "perfect simulation", is to sample the initial simulation state from the stationary regime. For most models this is hard to do, but, as we show, this is quite easy (from an implementation viewpoint) for the random trip model. Perfect simulation for the random waypoint was advocated and solved by Navidi and Camp in [13] who also give the stationary distribution (assuming location and speed are independent in the stationary regime, an issue later resolved in [10] using the Palm techniques in this paper).

\section{The Palm Calculus Framework}

The derivations in [13] involve long and sophisticated computations. We use a different approach, based on Palm calculus, a set of formulas that relate time averages to event averages. Palm calculus is now well established, but not widely used or even known in applied areas. For a quick overview of Palm calculus, see [11]; for 

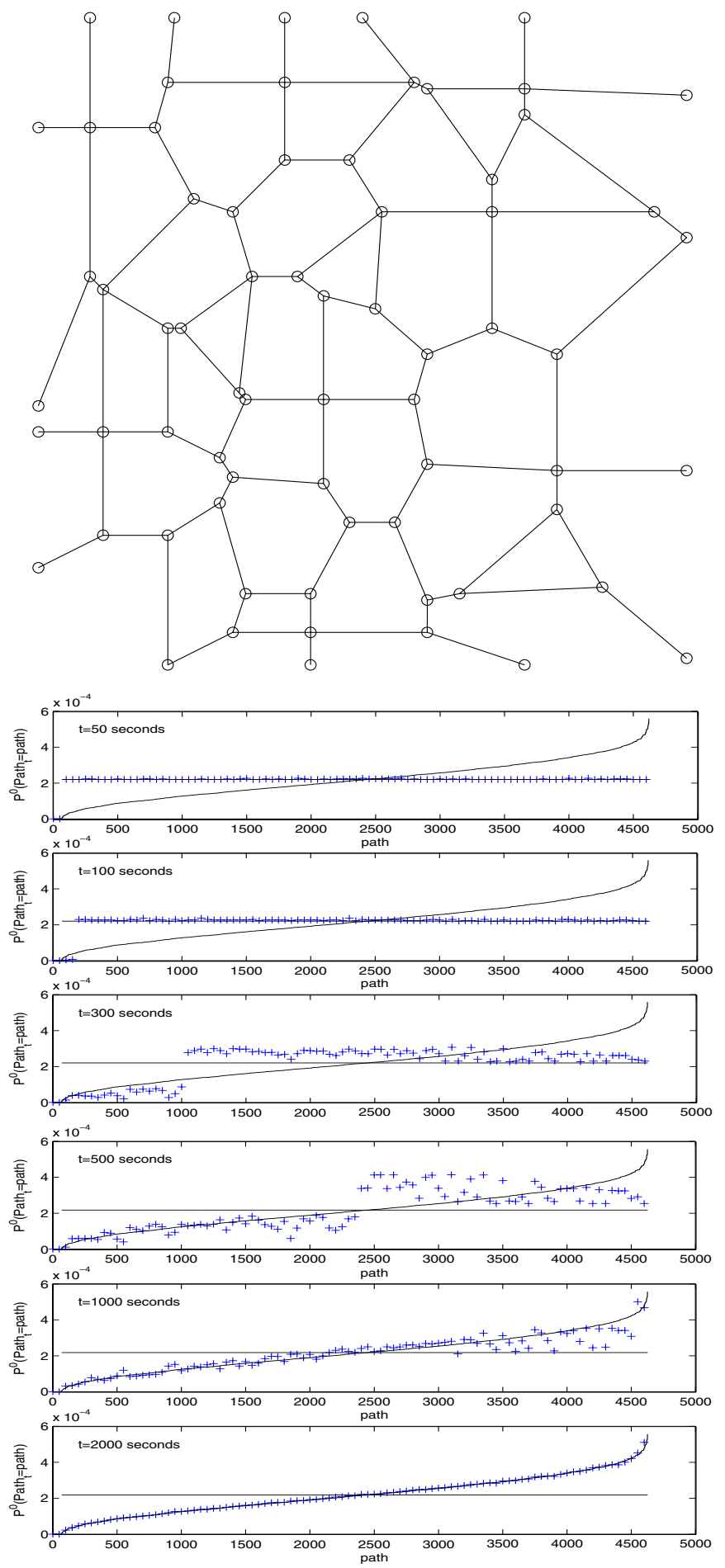

Fig. 1. Top: "Space Graph", a model proposed by Jardosh et al [9]. A mobile starts from a randomly chosen circle and goes along a shortest path towards another randomly chosen circle. Numerical speed is constant $=1.25 \mathrm{~m} / \mathrm{s}$. Bounding area $1 \mathrm{~km} \times 1 \mathrm{~km}$. Bottom: Probability distribution of the path used by a mobile at time $t$. Initially, the path is chosen uniformly among all possible paths. $x$ axis: path index, sorted by path length; $y$-axis: probability that this path is used at time $t$ for $t=50,100,300,500,1000,2000$ seconds of simulated time. Horizontal solid line: initial distribution; other solid line: time-stationary distribution. The transient lasts for a long time.

a full fledged theory, see [1]. This framework allows us to generalize the results in [13] to a broad class of models, as discussed next. Incidentally, even for the original random waypoint model, we provide new elements: a proof that a stationary regime exists when $v_{\min }>0$ and a sampling algorithm that, for complicated, non convex areas, does not require a priori computation of geometric integrals. More fundamentally, the Palm calculus framework allows us derive simple sampling algorithms for the generic random trip model - a task that would be formidable without this tool.

\section{Contributions of This Paper}

As a first step towards our goal, we give a model for independent mobiles (leaving group mobility models for further study). The model is called "random trip". In the absence of established properties of real mobility patterns, it is not yet clear today what the requirements on a mobility model should be [6]. We focus here on a model that is able to synthesize an a priori assumed mobile behaviour. This leads to examples such as city driving models ("Space Graph" [9], "City section" or "Hierarchical random waypoint", called "restricted random waypoint" in [3]), simple airplane circulation models ("Random Waypoint on Sphere"), or the special purpose "Fish in a Bowl" and "Swiss Flag". In some cases, it is desirable to assume that node location is uniformly distributed in steady-state; this is provided by the two "Random Walk" examples and by "Random Waypoint on a Sphere". We give a definition of the model and a non exhaustive list of examples in Section II.

Our main contributions are:

- a generic model and a framework to analyze it;

- a proven necessary and sufficient condition for a stationary regime to exist; a proof that when the stationary regime exists it is unique. This appears to be new even for the classical random waypoint;

- a generalization of random waypoint perfect simulation to non convex areas;

- a sampling algorithm that does not require the computation of geometric integrals;

- the proof that for three examples (random walk on a rectangle with wrapping or reflection, random waypoint on sphere) the node location is uniform. For the random walk examples, the steady state is essentially the same as the naive initialization (with uniform node placement) and there is no speed decay. In contrast, there is speed decay for random waypoint on a sphere.

We focus on perfect simulation and leave for a further paper the study of convergence (and its rate) to the stationary regime when it exists. Due to space limitations, we exhibit our results with most of the proofs delegated to the appendix of the full version [4]. A notation list is 
given in the next section. Related work is described at the end of the paper.

\section{A General Mobility Model}

We consider a generic family of models, defined by the following framework.

1) The domain $\mathcal{A}$ is a closed, bounded, connected (not necessarily convex) subset of $\mathbb{R}^{2}$ or $\mathbb{R}^{3}$.

2) $\mathcal{P}$ is a set of paths on $\mathcal{A}$. A path is a continuous mapping from $[0,1]$ to $\mathcal{A}$ that has a continuous derivative except maybe at a finite number of points (this is necessary to define the speed).

For $p \in \mathcal{P}, p(0)$ is the origin of $p, p(1)$ is its destination, and $p(u)$ is the point on $p$ attained when a fraction $u \in[0,1]$ of the path is traversed.

3) Trip Selection Rule: A trip is the combination of a duration and a path. The position $X(t)$ of the mobile at time $t$ is defined iteratively as follows. There is a set $T_{n} \in$ $\mathbb{R}, n \in \mathbb{Z}$ of transition instants, such that $T_{0} \leq 0<T_{1}<$ $T_{2}<\ldots$. At time $T_{n}$, a path $P_{n} \in \mathcal{P}$ and a trip duration $S_{n} \in$ $\mathbb{R}_{+}$are drawn according to some specified trip selection rule, specific to the model. The next transition instant is $T_{n+1}=T_{n}+S_{n}$ and the position of the mobile is $X(t)=$ $P_{n}\left(\frac{t-T_{n}}{S_{n}}\right)$ for $T_{n} \leq t \leq T_{n+1}$.

The trip selection rule is constrained to choose a path $P_{n}$ such that $P_{n}(0)=P_{n-1}(1)$. Further, we assume that, with probability 1 , the duration of the trip $S_{n}$ is positive (instantaneous transitions are not allowed).

4) Default Initialization Rule: at time $t=0$, the initial position, path, position on path, and remaining time until the next transition are drawn according to some specified default initialization rule. A common default rule considers that time 0 is the first transition instant $\left(T_{0}=0\right)$, and selects a path and trip duration according to the trip selection rule. However, as shown in Section I, this causes some problems, that are fixed by using the perfect simulation initialization rule, described in Section VI-B.

In addition, we do the following assumptions. They are essential for our model to be tractable, while supporting a very broad class of mobility models.

(H1) The trip selection rule depends on all past only through the current mobile location $M_{n}$ and the state of a Markov chain $I_{n}$. Further, $I_{n}$ depends on all past only through the last state $I_{n-1}$. More precisely, $I_{n}$ (the phase) is defined on some enumerable set $I$; it changes its value at transition instants $T_{n}$. Given that the phase selected at $T_{n}$ is $I_{n}=i$, and given the mobile location $M_{n}=m$ at time $T_{n}$, the path $P_{n}$ and the trip duration $S_{n}$ are drawn independently of all past until time $T_{n}$, with a distribution that may depend on $m$ and $i$ but not on $n$; the new value

\section{Notation List}

- $\mathcal{A}\left(\subset \mathbb{R}^{2}\right.$ or $\left.\mathbb{R}^{3}\right)$ : model domain, connected and bounded

- $d(m, n)$ length of shortest path in $\mathcal{A}$ from $m \in \mathcal{A}$ to $n \in \mathcal{A}$; if $\mathcal{A}$ is convex $d(m, n)=\|m-n\|$

- $T_{n}: n$th transition time, at which a new trip is defined

- $I_{n} \in I, M_{n} \in \mathcal{A}, P_{n} \in \mathcal{P}, S_{n} \in(0, \infty)$ : phase, starting point, path, trip duration for the $n$th trip

- $I(t) \in I, M(t) \in \mathcal{A}, P(t) \in \mathcal{P}, S(t) \in(0, \infty), X(t) \in \mathcal{A}$ : phase, starting point, path, trip duration for the trip used by mobile at time $t$, location at time $t . X\left(T_{n}\right)=M_{n}$ and if $T_{n} \leq t<T_{n+1}$ then $I(t)=I_{n}, M(t)=M_{n}$ and $S(t)=S_{n}$.

- $U(t) \in[0,1]$ : fraction of the current trip that was already traversed. Thus $U(t) S(t)$ is the time elapsed on the current trip and the location of the mobile at time $t$ is $X(t)=$ $p(U(t))$, with $p=P(t)$. We assume that the trip is done at a speed proportional to the default speed of the path, i.e. if $T_{n} \leq t<T_{n+1}$ then $U(t)=\frac{t-T_{n}}{T_{n+1}-T_{n}}=\frac{t-T_{n}}{S_{n}}$

- It follows that the speed vector of the mobile at a time $t$ that is not an end of trip is $\vec{V}(t)=\frac{1}{S(t)} \frac{\partial}{\partial u} p(U(t))$, with $p=P(t)$ and the numerical speed is $V(t)=\|\vec{V}(t)\|$.

- For some random variable $Z, \mathbb{E}^{0}(Z)$ is the "Palm expectation", which can be interpreted as the expectation, conditional to the event that a transition occurs at time 0 , when the system has a stationary regime. $\mathbb{E}^{0}$ denotes the event average viewpoint [1], [11]. For example $\mathbb{E}^{0}\left(S_{0}\right)=$ $\mathbb{E}^{0}(S(0))$ is the average trip duration; in contrast, when the system has reached steady-state, $\mathbb{E}(S(0))=\mathbb{E}(S(t))$ is the average duration of a trip, seen from an observer who samples the system at an arbitrary point in time. Both are usually different because the observer is more likely to sample a large trip duration.

of the chain $I_{n+1}$ is drawn in a way that depends only on $i$.

(H2) Either of the following is true:

(H2a) (i) The distribution of location $M_{n+1}$ at time $T_{n+1}$, conditional on all past phases until $T_{n}$, depends only on the phase $I_{n}$ and not on $n$. (ii) Moreover, there exist renewal points defined as follows. The chain of phases $I_{n}$ has a set of selected transitions $I^{*} \subseteq I^{2}$ such that the distribution of location $M_{n+1}$, given all past up to time $T_{n}$ and given $\left(I_{n}, I_{n-1}\right) \in I^{*}$, depends only on $I_{n}$;

or

(H2b) The distribution of location $M_{n}$ at time $T_{n}$ does not depend on $n, M_{n}$ is independent of $I_{n}$, and $\left(S_{n}, I_{n+1}\right)$ depends on all past only through $I_{n}$.

(H3) The Markov chain $I_{n}$ is positive recurrent. For example, this is true if $I$ is finite and the graph of the chain $I_{n}$ is connected.

As we show next, these assumptions are verified by a very large class of mobility models. 


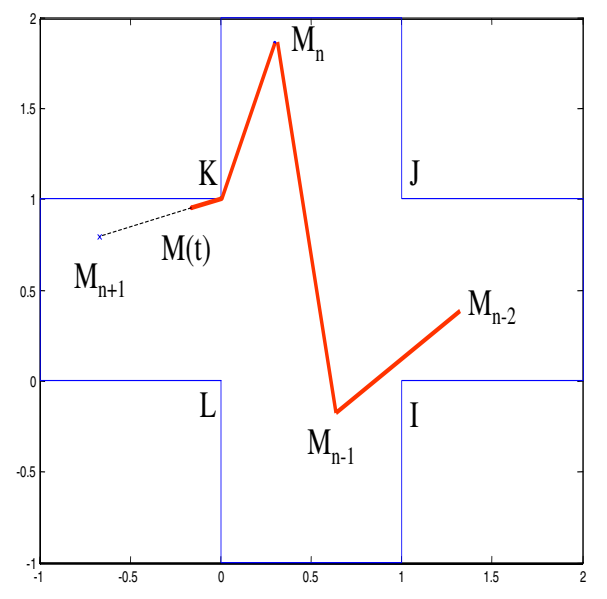

Fig. 2. Random Waypoint on a non convex domain (Swiss Flag). A trip is the shortest path inside the domain from a waypoint $M_{n}$ to the next. Waypoints $M_{n}$ are drawn uniformly in the domain. On the figure, the shortest path $M_{n}, M_{n+1}$ has two segments, with a breakpoint at $K$; the shortest paths $M_{n-1}, M_{n}$ and $M_{n-2}, M_{n-1}$ have one segment each. $M(t)$ is the current position.

\section{EXAMPLES}

We give a non exhaustive catalog of examples and show that they all fit in our framework.

\section{A. Classical Random Waypoint With Pauses.}

This is the classical random waypoint model. $\mathcal{A}$ is assumed to be convex ( $\mathcal{A}$ is a rectangle or a disk in [8], [6]). Paths are straight line segments: $p(u)=(1-u) m_{0}+$ $u m_{1}$ for the segment with endpoints $m_{0}$ and $m_{1}$. Pauses are special cases of paths, when endpoints are equal: $p(u)=m_{0}$. There are two phases $I=\{$ pause,move $\}$. At a transition instant, the trip selection rule alternates the phase from pause to move or vice versa. If the new phase is pause, the trip duration $S_{n}$ is picked according to the density $f_{\text {pause }}^{0}(s)$; the path $P_{n}$ is a pause at the current point. If the new phase is move, the trip selection rules picks a point $M_{n+1}$ at random uniformly in $\mathcal{A}$, and a numerical speed $V_{n}$ according to the density $f_{V}^{0}(v)$. A classical choice (uniform speed) is $f_{V}^{0}(v)=\frac{1}{v_{\max }-v_{\min }} 1_{\left\{v_{\min }<v<v_{\max }\right\}}$. The trip duration is then $S_{n}=\frac{\left\|M_{n+1}-M_{n}\right\|}{V_{n}}$ and the path $P_{n}$ is the segment $\left[M_{n}, M_{n+1}\right]$. The default initialization rule starts the model at the beginning of a pause, at a location uniformly chosen in $\mathcal{A}$.

The trip selection rule makes its choices only based on the current phase and location, thus H1 holds. The conditions (i) in $\mathrm{H} 2 \mathrm{a}$ are indeed true; the condition (ii) is true for the selected phase transitions pause $\rightarrow$ move. Hence, $\mathrm{H} 2 \mathrm{a}$ is verified. Further, the Markov chain $I_{n}$ alternates between the two states \{pause, move\}, thus hypothesis $\mathrm{H} 3$ is satisfied.

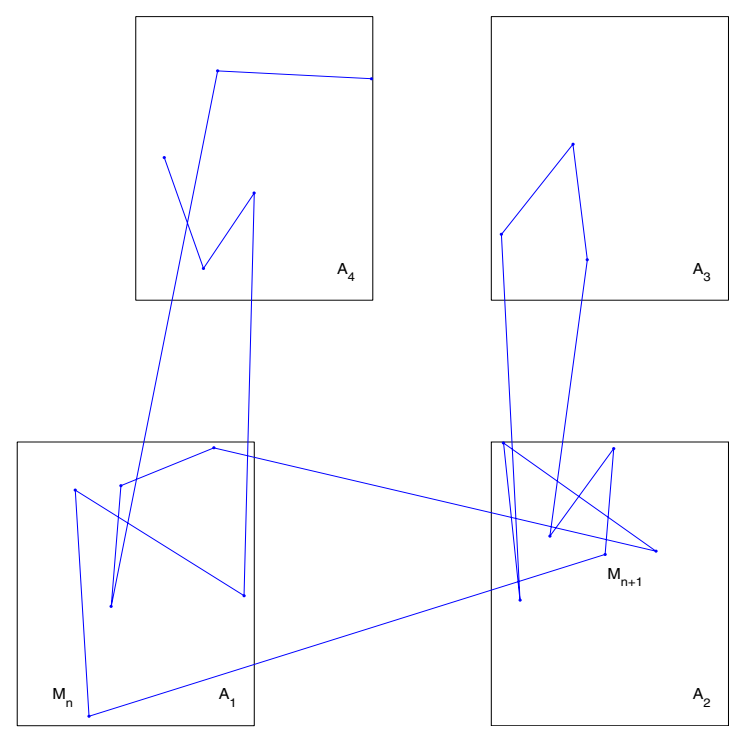

Fig. 3. Restricted random waypoint on a plane with four squares as subdomains. This model was introduced in [3] to simulate a wide-area routing protocol. It was used as an idealized view of four towns represented by squares. A mobile moves according to random waypoint within a square for a random number of visits and then picks a point uniformly at random in another randomly chosen square as a destination. The figure shows a sample path of the mobile movement. The speed on the trip is chosen according to a distribution that depends on the origin and destination squares.

This model is well known; its stationary properties are studied in [14], [8], [10]. However, even for this simple model our framework provides two new results: the proof of existence of a stationary regime, and a sampling algorithm for the stationary distribution over general areas that does not require the computation of geometric integrals.

\section{B. Random Waypoint on General Connected Domain.}

This is a variant of the classical random waypoint (Example III-A), where we relax the assumption that $\mathcal{A}$ is convex, but assume that $\mathcal{A}$ is a connected domain over which a uniform distribution is well defined. For two points $m, n$ in $\mathcal{A}$, we call $d(m, n)$ the distance from $m$ to $n$ in $\mathcal{A}$, i.e. the minimum length of a path entirely inside $\mathcal{A}$ that connects $m$ and $n . \mathcal{P}$ is the set of shortest paths between endpoints. The trip selection rule picks a new endpoint uniformly in $\mathcal{A}$, and the next path is the shortest path to this endpoint. If there are several shortest paths, one of them is randomly chosen according to some probability distribution on the set of shortest paths. The set of phases is $I=\{$ pause, move $\}$. This model fits in our framework for the same reasons as the former example.

1) Swiss Flag: The model is random waypoint on particular non-convex domain defined by the cross section as in Figure 2.

2) City Section: This is a special case of random waypoint on a non convex domain. The domain is the 


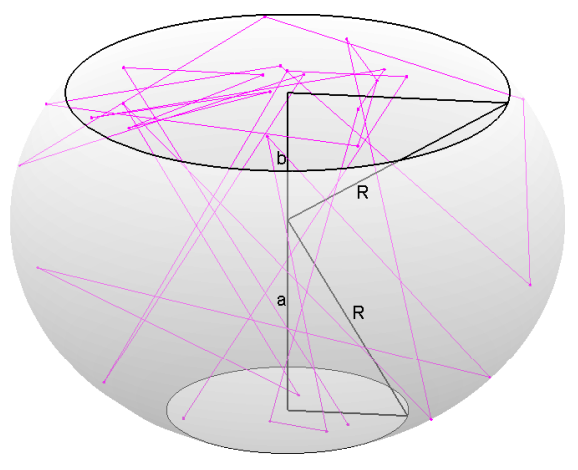

Fig. 4. Fish in a Bowl, a particular restricted random waypoint. $\mathcal{A}$ is the volume of the sphere comprised between two horizontal planes. Waypoints are in the subset $\mathcal{A}_{1}$ equal to the boundary of the spheric part of $\mathcal{A}$. For perfect sampling we do not need to know average Euclidean distance between two random points on the surface of the bowl. It suffices to know that the distance is at most $2 R$.

union of the segments defined by the edges of the space graph (e.g. Figure 1). Arbitrary numeric speeds can be assigned to edges of the graph. The "distance" from one location to another is the travel time.

\section{Restricted Random Waypoint.}

This model was originally introduced by Blažević et al [3]; see Figure 3 for a description. We define it slightly more generally as follows. As before, the domain $\mathcal{A}$ is connected, but not necessarily convex.

There are $L$ subdomains $\mathcal{A}_{\ell} \subset \mathcal{A}, \ell=1,2, \ldots, L$. (In the original model [3], $\mathcal{A}_{\ell}$ is a square, $\ell=1,2,3,4$, the subdomains are disjoint and $\mathcal{A}$ is the convex closure of $\bigcup_{\ell} \mathcal{A}_{\ell}$ ). The mobile executes a number of trips with endpoints in the same subdomain, then picks a new endpoint in some other subdomain $\ell^{\prime}$ and goes there along a shortest path. $\ell^{\prime}$ is chosen according to the transition matrix $Q\left(\ell, \ell^{\prime}\right)$, assumed to be irreducible and such that $Q(\ell, \ell)=0$. There is a pause between trips.

More precisely, a phase is a quadruple $I_{n}=\left(\ell, \ell^{\prime}, r, \phi\right)$ with $\ell, \ell^{\prime} \in\{1, \ldots, L\}$ (origin and destination subdomains), $r \in \mathbb{N}$ (residual number of trips in the same subdomain, including this one) and $\phi \in\{$ pause, move $\}$. If $\ell \neq \ell^{\prime}$ then $r=0$ else $r \geq 1$. The trip selection rule is executed at the end of a trip as follows. If $\phi=$ move then $\phi$ is set to pause, a pause is executed at the current location, for a duration drawn from a distribution that depends on the current subdomain, and $\ell, \ell^{\prime}, r$ are unchanged. Else $\phi$ is set to move, and $\ell, \ell^{\prime}, r$ are updated as follows. If $r \geq 1, r$ is decremented by 1 . If $r \geq 2$, $\ell$ and $\ell^{\prime}$ are unchanged (they must be equal). If $r=1$ (the previous trip was the last with endpoints in the current subdomain), $\ell^{\prime}$ is set to a new destination subdomain chosen according to the transition matrix $Q\left(\ell, \ell^{\prime}\right)$. If $r=0$

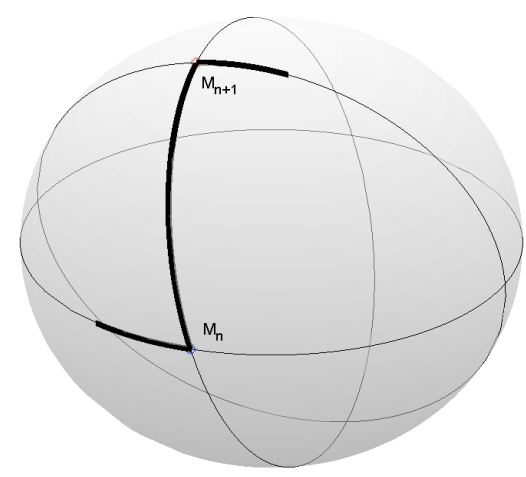

Fig. 5. Random waypoint on a sphere.

(the previous trip was between subdomains) $\ell$ is set to the value of $\ell^{\prime}$ and a new value of $r$ is drawn from a probability distribution that depends on $\ell^{\prime}$. Then a new endpoint is selected uniformly in $\mathcal{A}_{\ell^{\prime}}$ and the next trip is a shortest path from the current endpoint to this endpoint. For every trip, the numerical speed is selected according to a density that may depend on the origin and destination subdomains of the trip endpoints.

In addition to the model in Figure 3, we give two particular examples of the restricted random waypoint model.

1) Fish in a Bowl: The model is restricted random waypoint on the domain defined by the volume of the bowl, as in Figure 4. The waypoints are restricted to the subset $\mathcal{A}_{1}$ of the domain $\mathcal{A}$, where $\mathcal{A}_{1}$ is the set of the points on the bowl's surface (see Figure 4). The set of phases is $I=\{$ pause, move $\}$.

2) Space Graph: We defined this model in Section I. It is a special case of restricted random waypoint with $\mathcal{A}=$ the space graph and $\mathcal{A}_{1}=$ the set of vertices. Note that it differs from the City Section graph in that the waypoints are restricted to be vertices. The set of phases is $I=\{$ pause, move $\}$.

Note that all models III-A to III-C.2 and III-D are special cases of the restricted random waypoint, with $L=1, r=0$, and $\mathcal{A}_{1}=\mathcal{A}$ for examples III-A to IIIB.2, $\mathcal{A}$ a strict subset of $\mathcal{A}$ for examples III-C.1 and III-C.2. Note that the subdomains $\mathcal{A}_{\ell}$ may be convex as in Figure 3 or not as in Figure 4.

\section{Random Waypoint on Sphere.}

Here $\mathcal{A}$ is the unit sphere of $\mathbb{R}^{3} . \mathcal{P}$ is the set of shortest paths plus pauses. The shortest path between two points is the shortest of the arcs on the great circle that contains the two points. If the two points are on the same great circle diameter, the two arcs have same length (this occurs with probability 0 ). The trip transition rule picks 


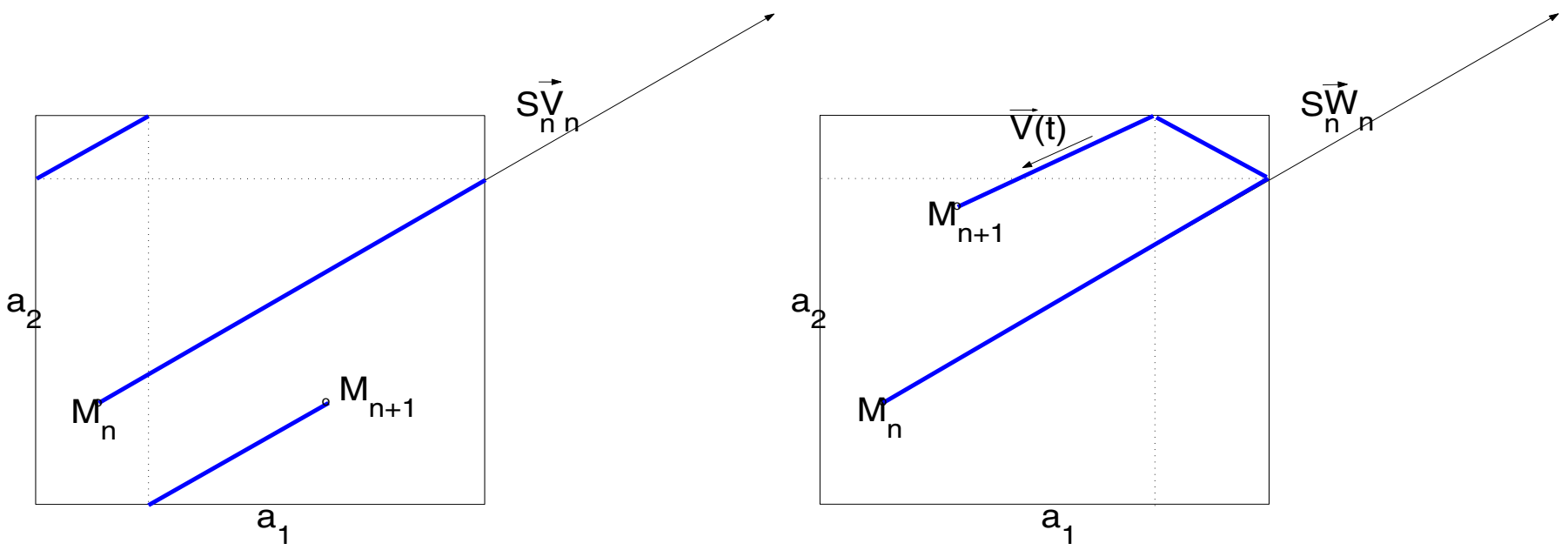

Fig. 6. Definition of Random Walk (Random Direction) with wrapping (left) or billiard-like reflection (right) at the edge of the domain.

a path endpoint uniformly on the sphere, and the path is the shortest path to it (if there are two, one is chosen with probability 0.5 ). The set of phases is $I=\{$ pause, move . The numerical speed is chosen independently. Initially, a point is chosen uniformly.

This model is in fact a special case of the random waypoint on a connected, non convex domain. However, we mention it separately as it enjoys special properties (the stationary location is uniform, unlike for the random waypoint models described earlier).

\section{E. Random Walk with Wrapping.}

This model is viewed as a random waypoint on a torus in [12]. It has similarity with the Random Direction in [6]. It is used primarily because of its simplicity: unlike for the random waypoint, the distribution of location and speed at a random instant are the same as at a transition instant, as we show later.

The domain $\mathcal{A}$ is the rectangle $\left[0, a_{1}\right] \times\left[0, a_{2}\right]$. Paths are wrapped segments, defined as follows. The trip selection rule chooses a speed vector $\vec{V}_{n}$ and a trip duration $S_{n}$ independently, according to some fixed distributions. Choosing a speed vector $\vec{V}_{n}$ is the same as choosing a direction of movement and a numerical speed. The mobile moves from the endpoint $M_{n}$ in the direction and at the rate given by the speed vector. When it hits the boundary of $\mathcal{A}$, say for example at a location $\left(x_{0}, a_{2}\right)$, it is wrapped to the other side, to location $\left(x_{0}, 0\right)$, from where it continues the trip (Figure 6). Let $w: \mathbb{R}^{2} \rightarrow \mathcal{A}$ be the wrapping function:

$$
\left(\begin{array}{l}
x \\
y
\end{array}\right) \mapsto w\left(\begin{array}{c}
x \\
y
\end{array}\right)=\left(\begin{array}{cc}
x & \bmod a_{1} \\
y & \bmod a_{2}
\end{array}\right) .
$$

The path $P_{n}$ (if not a pause) is defined by $\left(M_{n}, \vec{V}_{n}, S_{n}\right)$, such that $P_{n}(u)=w\left(M_{n}+u S_{n} \vec{V}_{n}\right)$. Note that wrapping does not modify the speed vector (Figure 6). After a trip, a pause time is drawn independent of all past from some fixed distribution. Initially, the first endpoint is chosen uniformly in $\mathcal{A}$. As we show next, this implies that all endpoints are in turn uniformly distributed (when sampled at transition instants).

This model obviously satisfies assumptions $\mathrm{H} 1$ and H3 with set of phases $I=\{$ pause, move $\}$. We now show that it satisfies $\mathrm{H} 2$.

Lemma 1: Let $X$ be a random point, uniformly distributed in $\mathcal{A}=\left[0, a_{1}\right] \times\left[0, a_{2}\right] \times \ldots\left[0, a_{d}\right]$. For any non random vector $\vec{v} \in \mathbb{R}^{d}$, the distribution of $w(X+\vec{v})$ is also uniform in $\mathcal{A}$.

Theorem 1: The distribution of points $M_{1}, M_{2}, \ldots$, is uniform in $\mathcal{A}$.

Proof. $M_{0}$ is uniform by assumption. By Lemma 1, the distribution of $M_{1}$ is also uniform, and recursively, so is the distribution of $M_{n}$.

This shows H2b. Note that this is true regardless of the distribution with which $\vec{V}_{n}$ and $S_{n}$ are chosen.

\section{F. Random Walk with Reflection.}

This is similar to example III-E, but with billiard-like reflections instead of wrapping (Figure 6). It enjoys some of the same final simplicity, but the intermediate steps are more elaborate. The definition is identical to example III-E, with the three following differences:

1) The wrapping function is replaced by the billiard reflection function $b: \mathbb{R}^{2} \rightarrow \mathcal{A}$, defined by

$$
\left(\begin{array}{c}
x \\
y
\end{array}\right) \mapsto b\left(\begin{array}{c}
x \\
y
\end{array}\right)=\left(\begin{array}{l}
a_{1} b_{1}\left(\frac{x}{a_{1}}\right) \\
a_{2} b_{1}\left(\frac{y}{a_{2}}\right)
\end{array}\right),
$$

where $b_{1}: \mathbb{R} \rightarrow[0,1]$ is the 2-periodic function:

$$
b_{1}(x)=|x|, \text { for }-1 \leq x \leq 1 .
$$


2) Unlike the wrapping function, the billiard reflection may alter the speed vector (Figure 6). Therefore we differentiate the unreflected speed vector $\vec{W}_{n}$ from the instantaneous speed vector $\vec{V}(t)$ at time $t$. The path $P_{n}$ (if not a pause) is defined by $\left(M_{n}, \vec{W}_{n}, S_{n}\right)$, such that $P_{n}(u)=b\left(M_{n}+u S_{n} \vec{W}_{n}\right)$.

3) We assume that the distribution of the unreflected speed vector $\vec{W}_{n}$ chosen by the trip selection rule has a density and is completely symmetric. We say that a random vector $(X, Y)$ has a completely symmetric distribution iff $(-X, Y)$ and $(X,-Y)$ have the same distribution as $(X, Y)$. This is true for example if the direction of $\vec{W}$ is uniformly chosen on the unit circle, or if the two coordinates of $\vec{W}$ are independent and have even distributions.

This model obviously satisfies assumptions $\mathrm{H} 1$ and $\mathrm{H} 3$ with set of phases $I=\{$ pause, move $\}$. It also satisfies $\mathrm{H} 2$ :

Lemma 2: Let $X$ be a random point, uniformly distributed in $\mathcal{A}=\left[0, a_{1}\right] \times\left[0, a_{2}\right] \times \ldots\left[0, a_{d}\right]$. Let $\vec{V}$ be a random vector in $\mathbb{R}^{d}$ with a completely symmetric density. The distribution of the reflection $b(X+\vec{V})$ is also uniform in $\mathcal{A}$.

Proof. Follows from Lemma 5 in Section VII.

Theorem 2: The distribution of points $M_{1}, M_{2}, \ldots$, is uniform in $\mathcal{A}$.

Proof. Similar to Theorem 1, using Lemma 2.

\section{EXISTENCE AND UNIQUENESS OF STATIONARY DISTRIBUTION}

Theorem 3: With the model defined in Section II, there is a time-stationary regime if and only if the expected trip duration $\mathbb{E}^{0}\left(S_{0}\right)$ is finite. If it exists, the stationary regime is unique.

Proof of the theorem in Appendix [4] is outlined as follows. First, under assumptions H1-H3, it follows that there exists a unique stationary distribution for the mobility state embedded at trip transition instants (aka event-stationary distribution). Second, we show that the so called Slivnyak's conditions hold [1], which guarantee existence of a time-stationary distribution. Lastly, whenever a time-stationary distribution exists, its uniqueness follows from the Palm inversion formula [1].

Corollary 1: For examples III-A to III-D, there is a stationary regime if and only if the pause time and inverse speed (sampled at a transition) have a finite expectation. For examples III-E and III-F the condition is that the pause time and trip duration (sampled at a transition) have a finite expectation.

Comment. These conditions are known to be necessary for the classical random waypoint to be "harmless".
However, it appears to be the first time that the link to the existence of a stationary regime is made rigorously.

\section{TIme Stationary Distributions}

For a perfect simulation, all we need is to sample from the time stationary distribution of the process state. The state of the process is the phase $(I(t)$, the path $P(t)$, the trip duration $S(t)$ and fraction of time elapsed on the trip $U(t)$. In this section we derive the fundamental relation between the parameters of the random trip model and its stationary distribution. In the next section we apply it to the various examples introduced earlier.

Theorem 4: Assume the condition for existence and uniqueness of a stationary distribution in Section IV is satisfied. The time stationary distribution of the process state at an arbitrary time $t$ is defined as follows.

1) Phase:

$$
\mathbb{P}(I(t)=i)=\frac{\pi^{0}(i) \bar{\tau}_{i}}{\sum_{j} \pi^{0}(j) \bar{\tau}_{j}}
$$

where $\bar{\tau}_{i}=\mathbb{E}^{0}\left(S_{0} \mid I_{0}=i\right)$ is the mean trip duration for phase $i$.

2) Path and trip duration, given the phase:

$$
\begin{aligned}
& d \mathbb{P}(P(t)=p, S(t)=s \mid I(t)=i) \\
& \quad=\frac{s}{\bar{\tau}_{i}} d \mathbb{P}^{0}\left(P_{0}=p, S_{0}=s \mid I_{0}=i\right) .
\end{aligned}
$$

3) Fraction of time elapsed on the trip:

$U(t)$ is independent of $(I(t), P(t), S(t))$ and is uniform on $[0,1]$.

Note that the factor $1 / \sum_{i} \pi^{0}(i) \bar{\tau}_{i}$ in item 1 is precisely the intensity of the point process of trip transitions [1]. Special Case. In many examples (III-A to III-C.2 and III-D) the set of phases is reduced to $\{$ pause, move $\}$ and the model alternates between these two. Then $\pi^{0}(i)=0.5$ for $i=$ pause or move and item 1 simplifies to $P(I(t)=$ pause $)=\frac{\bar{\tau}_{\text {pause }}}{\bar{\tau}_{\text {pause }}+\bar{\tau}_{\text {move }}}$ and $P(I(t)=$ move $)=\frac{\bar{\tau}_{\text {move }}}{\bar{\tau}_{\text {pause }}+\bar{\tau}_{\text {move }}}$.

\section{Application to EXAmples III-A to III-C}

In all of this section, we assume that the condition for stationarity in Section IV is satisfied. We focus on restricted random waypoint on general connected area, since examples III-A to III-C are special cases of it.

\section{A. Time Stationary Distributions}

A direct application of the Theorem 4 gives the time stationary distribution of the process. Due to its description complexity, we give it in three pieces, in the following theorems. Special notation local to this section is given below. 


\section{Notation Used in Section VI}

- $Q\left(\ell, \ell^{\prime}\right)$ : probability that next subdomain is $\mathcal{A}_{\ell^{\prime}}$ given current subdomain is $\mathcal{A}_{\ell}$, with $Q(\ell, \ell)=0 . q^{*}(\ell)$ is the unique stationary probability of $Q\left(q^{*} Q=q^{*}\right)$.

- For $r \in \mathbb{N}, F_{\ell}(r)$ is the probability that the number of consecutive sojourns in subdomain $\mathcal{A}_{\ell}$ is $\geq r . \bar{R}_{\ell}=\sum_{r} F_{\ell}(r)$ is the average number of consecutive sojourns in subdomain $\mathcal{A}_{\ell}$.

- $\bar{\Delta}_{\ell, \ell^{\prime}}$ is the average distance in $\mathcal{A}$ for two points chosen uniformly in $\mathcal{A}_{\ell}$ and $\mathcal{A}_{\ell^{\prime}}$. $\Delta_{\ell, \ell^{\prime}}$ is some upper bound on the distance in $\mathcal{A}$ between two points in $\mathcal{A}_{\ell}$ and $\mathcal{A}_{\ell^{\prime}}$.

- $f_{V \mid i}^{0}(v)$ is the Palm (= at a transition instant) distribution of speed, given that phase is $i=\left(\ell, \ell^{\prime}, r\right.$, move $) ; \omega_{\ell, \ell^{\prime}}=$ $\mathbb{E}^{0}\left(\frac{1}{V_{0}} \mid I_{n}=\left(\ell, \ell^{\prime}, r\right.\right.$, move $\left.)\right)$ is the event average of the inverse of the speed chosen for a trip from subdomain $\mathcal{A}_{\ell}$ to $\mathcal{A}_{\ell^{\prime}}$. We have $\omega_{\ell, \ell^{\prime}}=\int_{0}^{\infty} \frac{1}{v} f_{V \mid \ell, \ell^{\prime}, r, \text { move }}(v) d v$, assumed to be independent of $r$.

- $f_{S \mid i}^{0}(s)$ is the Palm (= at a transition instant) distribution of pause time, given that phase is $i=\left(\ell, \ell^{\prime}, r\right.$, pause $) ; \tau_{\ell, \ell^{\prime}}=$ $\mathbb{E}^{0}\left(S_{0} \mid I_{0}=\left(\ell, \ell^{\prime}, r\right.\right.$, pause $\left.)\right)$ is the average pause time that follows a trip from subdomain $\mathcal{A}_{\ell}$ to $\mathcal{A}_{\ell^{\prime}}$. We have $\tau_{\ell, \ell^{\prime}}=$ $\int_{0}^{\infty} s f_{S \mid \ell, \ell^{\prime}, r \text {,pause }}(s) d s$, assumed to be independent of $r$.

The first theorem generalizes known statements for the classical random waypoint (Example III-A) [15], [14]. It relates the time average speed to the distribution of the speed selected at a waypoint, and contains an exact representation of the time stationary distribution of location.

Theorem 5: Under the time stationary distribution, conditional to phase $I(t)=i=\left(\ell, \ell^{\prime}, r\right.$, move $)$ :

1) The numerical speed is independent of the path and the instantaneous location of the mobile at time t. Its density is

$$
f_{i}(v)=\frac{C_{i}}{v} f_{V \mid i}^{0}(v)
$$

where $f_{V \mid i}^{0}(v)$ is the density of the numerical speed sampled at a transition instant and $C_{i}$ is a normalizing constant.

2) The path endpoints $(P(t)(0), P(t)(1))$ have a joint density over $\mathcal{A}_{\ell} \times \mathcal{A}_{\ell^{\prime}}$ given by

$$
\begin{aligned}
& d \mathbb{P}\left(P(t)(0)=m_{0}, P(t)(1)=m_{1} \mid I(t)=i\right) \\
& \quad=K_{\ell, \ell^{\prime}} d\left(m_{0}, m_{1}\right)
\end{aligned}
$$

where $K_{\ell, \ell^{\prime}}$ are normalizing constants and $d()$ is the distance in $\mathcal{A}$.

3) The distribution of $X(t)$, given $P(t)(0)=p$ and $P(t)(1)=n$, is uniform on the segment $[p, n]$.

Proof. Apply Theorem 4 to obtain the joint distribution of the path, location and speed $V(t)$, by noting that $V(t)=d(P(t)(0), P(t)(1)) / S(t)$.
Comment 1. As we show later, there is no need to know the value of the constants $K_{\ell, \ell^{\prime}}$ to use the theorem in a simulation. ${ }^{1}$

Comment 2. The distribution of path endpoints $P(t)(0)$ and $P(t)(1)$ is not uniform, and the two endpoints are correlated (they tend to be far apart), contrary to what happens when sampled at transition instants. This was found already for Example III-A in [13].

Comment 3. The relation between time stationary and event stationary distribution of speed is sometimes interpreted as "speed decay" since it is more likely to produce low speed values than the density $f_{i}^{0}(v)$. If one desires a uniform speed distribution in time average, then the density of speed at transition instants should be $f_{i}^{0}(v)=K_{i}^{\prime} v 1_{\left\{v_{\min }<v<v_{\max }\right\}}$. Note that such a speed distribution satisfies the stability condition in Section IV even if $v_{\min }=0$.

Theorem 6: Under the time stationary distribution, conditional to phase $I(t)=i=\left(\ell, \ell^{\prime}, r\right.$,pause $)$ :

1) the location $X(t)$ and the time $R(t)$ until end of pause are independent

2) $X(t)$ is uniform in $\mathcal{A}_{\ell^{\prime}}$

3) $R(t)$ has density

$$
f_{i}(r)=\frac{1}{\bar{\tau}_{i}} \int_{r}^{\infty} f_{S \mid i}^{0}(s) d s
$$

where $f_{S \mid i}^{0}(s)$ is the density of the pause time selected at a transition.

Proof. Similar to (but simpler than) Theorem 5.

We next show time-stationary distribution for phase, but only for the special case $L=1$, i.e. one subdomain. The general case for arbitrary $L$ bears some notational complexity and is for this reason deferred to Appendix [4].

Theorem 7: The time stationary distribution of phase $\pi$ is given by is

$$
\pi(\text { pause })=\frac{\tau_{\text {pause }}}{\tau_{\text {pause }}+\bar{\Delta} \omega}
$$

and $\pi$ (move $)=1-\pi$ (pause $)$, where $\tau_{\text {pause }}$ is the average pause time, $\bar{\Delta}$ the average distance in $\mathcal{A}$ between two points in $\mathcal{A}_{1}$, and

$$
\omega=\mathbb{E}^{0}\left(\frac{1}{V_{0}} \mid I_{0}=\text { move }\right) .
$$

\footnotetext{
${ }^{1}$ However, in the special case of convex domains where $d(m, n)$ is the usual Euclidean distance, it is worth noting that there are known formulae: $K_{\ell, \ell^{\prime}}^{-1}=\operatorname{vol}\left(\mathcal{A}_{\ell}\right) \operatorname{vol}\left(\mathcal{A}_{\ell}^{\prime}\right) \bar{\Delta}_{\ell, \ell^{\prime}}$ where $\operatorname{vol}\left(\mathcal{A}_{\ell}\right)$ is the area or volume of $\mathcal{A}_{\ell}$ (in square or cubic meters) and $\bar{\Delta}_{\ell, \ell^{\prime}}$ is the average distance in $\mathcal{A}$ between two points drawn uniformly in $\mathcal{A}_{\ell}$ and $\mathcal{A}_{\ell^{\prime}}$. For $\ell=\ell^{\prime}$ and $\mathcal{A}_{\ell}=$ a square of a size $a, K_{\ell, \ell}^{-1} \approx 0.5214 a^{5}$; for a disk of radius $a, K_{\ell, \ell}^{-1} \approx 0.9054 \pi^{2} a^{5}$ [8]. For an arbitrary case, it is generally not possible to obtain either $\operatorname{vol}\left(\mathcal{A}_{\ell}\right)$ or $\bar{\Delta}_{\ell, \ell^{\prime}}$ in closed form, but $K_{\ell, \ell^{\prime}}^{-1}$ can be obtained directly by Monte Carlo simulation.
} 
is the event average of the inverse of the speed.

As with Theorem 5, we show later that we do not need to know $\bar{\Delta}$ to use this theorem for sampling. The special case of one sub-domain accomodates examples III-A, III-B.1, III-B.2, III-C.1, III-C.2, and III-D.

\section{B. Perfect Simulation Without Computing Geometric In- tegrals}

A straightforward application of the previous section poses the problem of how to sample $\left(m_{0}, m_{1}\right)$ from the density in Theorem 5. Further, in order to sample the phase in Theorem 7 one needs to compute the geometric integrals $\bar{\Delta}_{\ell, \ell^{\prime}}$; for simple cases $\left(L=1\right.$ and $\mathcal{A}_{1}$ is a rectangle or disk) there exist closed forms, as mentioned in Comment 1 after Theorem 5. Otherwise, one needs to compute them offline by Monte Carlo simulation. For cases like Figure 3, this is time consuming (see an analysis in Appendix [4]). There is generally more efficient procedure, which avoids computing the geometric integrals when they are not known. The solution of these two problems is based on the following lemma.

1) Rejection Sampling Lemma: Let $(J, Y)$ be a random vector, where $J$ is in a discrete set $\mathcal{I}$ and $Y \in \mathbb{R}^{d}$. Assume that $\mathbb{P}(J=j)=\lambda \mu(j) \varpi_{j}$ and the distribution of $Y$ conditional to $J=j$ has a density $\frac{f_{j}(y)}{\varpi_{j}}$ The problem is to sample from $(J, Y)$ without having to compute the normalizing constants of the densities $\varpi_{j}$ for all $j$.

Assume we know factorizations of the form $f_{j}(y)=$ $k_{j}(y) g_{j}(y)$ where $g_{j}(y)$ is a probability density ${ }^{2}$ Assume also that we know upper bounds $\kappa_{j}$ such that $0 \leq k_{j}(y) \leq$ $\kappa_{j}$.

Lemma 3: Let $\mathrm{v}$ be the probability on $\mathcal{I}$ defined by: if $\varpi_{j}$ is known $v(j)=\alpha \mu(j) \varpi_{j}$ else $v(j)=\alpha \mu(j) \kappa_{j}$, where $\alpha$ is a normalizing constant, defined by the condition $\sum_{j} v(j)=1$. The following algorithm draws a sample from $(J, Y)$ :

\section{do forever}

draw $j$ with probability $\mathrm{v}(j)$

if $\bar{\varpi}_{j}$ is known

draw y from the density $f_{j}(y) / \varpi_{j}$; leave

else

draw $y$ from the density $g_{j}(y)$

draw $U \sim \operatorname{Unif}\left(0, \kappa_{j}\right)$

if $U \leq \frac{k_{j}(y)}{\kappa_{j}}$ leave

\section{end do}

Comment. The lemma follows by the structure of the distribution of $J$ and conditional density of $Y$. The structure is: $\mathbb{P}(J=j)$ is proportional to $\bar{\omega}_{j}$, while the

\footnotetext{
${ }^{2}$ That is, $\int g_{j}(y) d y=1$, or in other words there is no normalizing constant to compute for $g_{j}(y)$.
}

conditional density of $Y$, given $J=j$, is inversely proportional to $\bar{\omega}_{j}$. By this structure, twisting the original distribution of $J$ and conditional density of $Y$, by replacing $\bar{\omega}_{j}$ with $\kappa_{j}$, indeed results in the original joint density of $(J, Y)$. The lemma is a general result. However, it may be helpful to note that the general form was suggested by particular distributions in Theorem 4 . Therein, phase $I(t)$ acts the role of $J$, while $(P(t), S(t), U(t))$ acts the role of $Y$.

2) The Sampling Method: The following theorem gives the sampling method. The details for the general case have some description complexity, and is for this reason deferred to Appendix [4]. We show all details here for the case $L=1$.

Theorem 8: (Perfect Simulation of Restricted Random Waypoint) The following algorithm draws a sample of the time stationary state of the restricted random waypoint:

1) Sample a phase $I(t)=i=\left(\ell, \ell^{\prime}, r, \phi\right)$ from the algorithm in Figure 7 (simple case) or in Appendix [4] (general case).

2) If $\phi=$ pause

- Sample a time $t$ from the distribution with density $f_{i}(t)=1 / \bar{\tau}_{i} \int_{t}^{\infty} f_{S \mid i}^{0}(s) d s$.

- Sample a point $M$ uniformly in $\mathcal{A}_{\ell^{\prime}}$.

- Start the simulation in pause phase at location $M$ and schedule the end of pause at $t$.

3) If $\phi=$ move

- Sample a speed $v$ from the distribution with density proportional to $\frac{1}{v} f_{V \mid i}^{0}(v)$.

- Set $M_{0}, M_{1}$ to the value returned by the algorithm in Figure 7 (simple case) or in Appendix [4] (general case).

- Sample u uniformly in $(0,1)$.

- Start the simulation in move phase, with initial position $(1-u) M_{0}+u M_{1}$, next trip endpoint $=M_{1}$, and speed $=v$.

Note that the algorithm in Figure 7 solves both problems mentioned in the introduction of this section. If $\bar{\Delta}$ is known with little computational cost (i.e. when $\mathcal{A}$ is a rectangle or a disk) it is always preferable to use the former case (" $\bar{\Delta}$ is known"). Else there are two options: (1) compute $\bar{\Delta}$ offline by Monte-Carlo simulation and use the case " $\bar{\Delta}$ is known", or (2) use the case (" $\bar{\Delta}$ is not known"). Apart from unusually long simulation campaigns with the same model, the optimal choice, in terms of number of operations is to use the latter case (see Appendix [4]). Furthermore, using the latter case simplifies the overall simulation code development. Figure 8 illustrate the sampling method on Examples IIIB to III-C. 

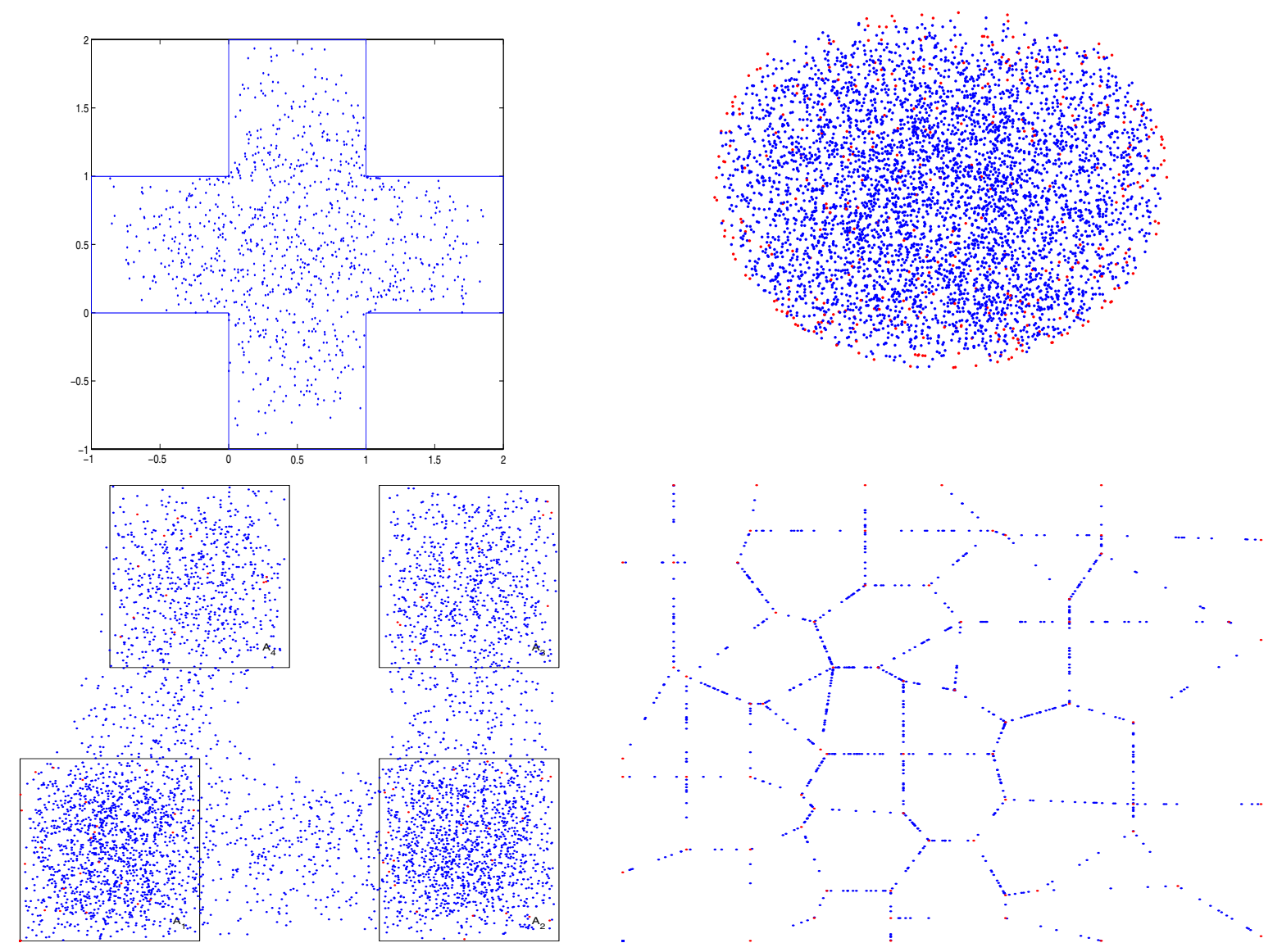

Fig. 8. Perfect sampling of node position from time-stationary distribution for swiss flag (1000 samples), fish in a bowl (5000 samples), four-town-restricted random waypoint (5000 samples) and space graph (10000 samples). Densities are not uniform, with bias towards central areas and interior corner points.

\section{ViI. Application to Examples III-D to III-F}

In all of this section, we assume that the condition for stationarity in Section IV is satisfied.

\section{A. Random Waypoint on Sphere (Example III-D)}

This model is a special case of restricted random waypoint over a non convex area, with $L=1$ and $\mathcal{A}_{1}=\mathcal{A}$. Thus all findings of Section VI apply, in particular, the time stationary speed is independent of location and is given by Theorem 5 .

Theorem 9: For the random waypoint on a sphere, the time stationary distribution of the mobile location is uniform.

Proof. Apply Theorem 5. The distribution of $X(t)$ is invariant under any rotation of the sphere around an axis that contains the center of the sphere, and any distribution that has such an invariance property must be uniform.

Note that, with the same argument, we can show that, given we are in a move phase, the time stationary distribution of each path endpoint (previous and next) separately is also uniform, but the two endpoints are correlated (it is more likely that they are far apart). This is because, from Theorem 5, a typical path seen in time average is drawn with a probability proportional to path length. This implies that, though the time stationary distribution of points is uniform, it is not sufficient for perfect simulation to draw an initial position uniformly on the sphere and start as if it would be a path endpoint (we need in addition to sample a path and where on path according to Theorem 5).

\section{B. Random Walk with Wrapping (Example III-E)}

Let $f_{\text {pause }}^{0}(t)$ [resp. $f_{\text {move }}^{0}(t)$ ] be the density of the pause [resp. move] duration, sampled at a transition time. Both densities are model parameters. Also let $\bar{\tau}_{\text {pause }}, \bar{\tau}_{\text {move }}$ be the corresponding averages (thus for example $\bar{\tau}_{\text {pause }}=$ $\mathbb{E}^{0}\left(S_{0} \mid I_{0}=\right.$ pause $\left.)=\int_{0}^{\infty} t f_{\text {pause }}^{0}(t) d t\right)$. Finally, let $f_{\vec{V}}^{0}(\vec{v})$ be the density of the distribution of the speed vector (sampled at trip endpoints). 


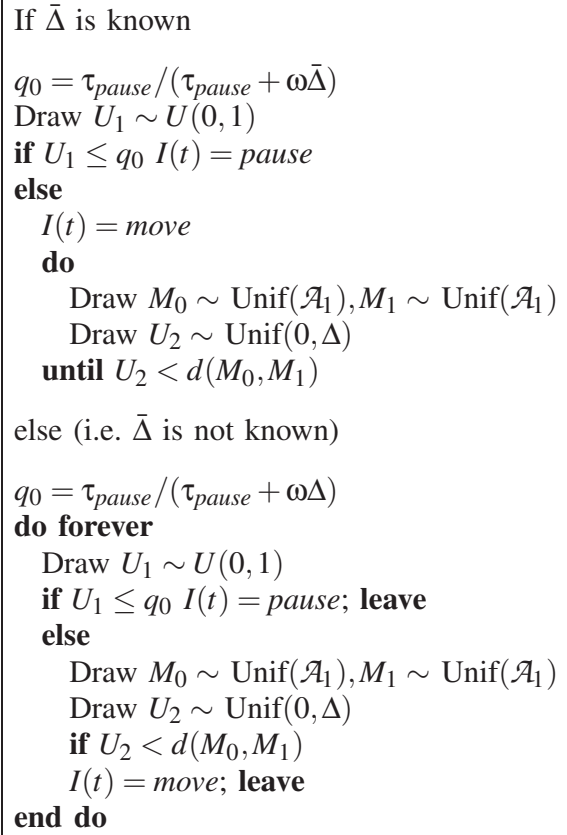

Fig. 7. Sampling algorithm for restricted random waypoint with $L=$ 1 , supporting both cases where the average distance between points in $\mathcal{A}_{1}$ is known or not. $\tau_{\text {pause }}$ is the average pause time, $\bar{\Delta}$ the average distance in $\mathcal{A}$ between two points in $\mathcal{A}_{1}, \Delta$ an upper bound on the distance in $\mathcal{A}$ between two points in $\mathcal{A}_{1}$ and $\omega=\mathbb{E}^{0}\left(1 / V_{0} \mid I_{0}=\right.$ move $)$

Theorem 10: For random walk with wrapping, under the time stationary distribution:

1) the process state at time $t$ is fully described by the phase $I(t)$, the location $X(t)$, the speed vector $\vec{V}(t)$ $(=\overrightarrow{0}$ if phase $=$ pause $)$ and the residual time until end of trip $R(t)$

2) the location $X(t)$ is uniform

3) $P(I(t)=$ pause $)=\frac{\bar{\tau}_{\text {pause }}}{\bar{\tau}_{\text {pause }}+\bar{\tau}_{\text {move }}}$ and $P(I(t)=$ move $)=$ $\frac{\bar{\tau}_{\text {move }}}{\bar{\tau}_{\text {pause }}+\bar{\tau}_{\text {move }}}$

4) conditional to phase=pause:

- the residual pause duration $R(t)$ has density $f_{\text {pause }}(r)=1 / \bar{\tau}_{\text {pause }} \int_{r}^{\infty} f_{\text {pause }}^{0}(s) d s$

- $X(t)$ and $R(t)$ are independent

5) conditional to phase $=$ move:

- $\vec{V}(t)$ has density $f_{\vec{V}}^{0}(\vec{v})$

- the residual trip duration $R(t)$ has density $f_{\text {move }}(r)=1 / \bar{\tau}_{\text {move }} \int_{r}^{\infty} f_{\text {move }}^{0}(s) d s$

- $X(t), \vec{V}(t)$ and $R(t)$ are independent

Thus, contrary to random waypoint on sphere, perfect simulation of this model is very simple. Pick a phase in proportion to the average time spent in the phase. Pick a point and, for move phase, a speed vector as if at a transition point, and pick a remaining trip duration according to the general formula for the density of the time until next transition, in any stationary system. Also, there is no speed decay [16] as with random waypoint on a sphere.

\section{Random Walk with Reflection (Example III-F)}

There is a similar result for random walk with billiard reflection, but its proof if more elaborate. We use continue with the same notation, with the difference that the instantaneous speed $\vec{V}(t)$ may differ from the unreflected speed $\vec{W}_{n}$ chosen at the beginning of the trip. Let $f_{\vec{W}}^{0}(\vec{w})$ be the density of the distribution of the non reflected speed vector (sampled at trip endpoints).

The following lemma expresses that, in order to continue a path from an intermediate point $m$ it is not needed to know the unreflected speed vector, the instantaneous speed is enough (proof in Appendix [4]):

Lemma 4: For any non random point $m \in \mathcal{A}$ and vector $\vec{v} \in \mathbb{R}^{2}: b(m+\vec{v})=b\left(b(m)+\nabla b_{m+\vec{v}} \vec{v}\right)$.

The following lemma says that, at the end of trip that starts from a uniform point $M$ and a completely symmetric initial speed vector $\vec{W}$, the reflected destination point $M^{\prime}$ and speed vector $\vec{W}^{\prime}$ are independent and have same distribution as initially (proof in Appendix [4]).

Lemma 5: Let $M$ be a random point, uniform in $\mathcal{A}$. Let $\vec{W}$ be a random vector in $\mathbb{R}^{2}$ independent of $M$ and with completely symmetric distribution under reflections. Let $M^{\prime}=b(M+\vec{W})$ and $\vec{W}^{\prime}=\nabla b_{M+\vec{W}} \vec{W} \cdot M^{\prime}$ and $\vec{W}^{\prime}$ are independent and have the same distribution as $M$ and $\vec{W}$.

Theorem 11: For the random walk with reflection, the same holds as in Theorem 10 after replacing $f_{\vec{V}}^{0}(\vec{v})$ by $f_{\vec{W}}^{0}(\vec{v})$ in the first bullet of item 5 .

Proof. Item 1 follows from Lemma 4. The rest follows from Theorem 4 and Lemma 5, in a similar way as for Theorem 10.

Remark. The location $X(t)$ and the path $M(t), \vec{W}(t), S(t)$ are not independent. For example, given that the unreflected speed vector is $\vec{W}(t)=\left(0.5 a_{1}, 0\right)$ and the trip duration is $S(t)=1$, it is more likely that $X(t)$ is in the second right half of the rectangle. However, independence is true if, instead of the path descriptor, we take as simulation state the current position, speed and time to next endpoint, as justified by the theorem. Perfect simulation of this random walk is similar to the random walk with wrapping.

\section{RELATED WORK}

For a survey of existing mobility models, see the work by Camp, Boleng, and Davies [6] and the references therein. Bettstetter, Hartenstein, and Pérez-Costa [8] studied the time-stationary distribution of a node position for classical random-waypoint model. They observed that the time-stationary node position is non-uniform and it has more mass in the center of a rectangle. A similar problem has been further studied by Bettstetter, Resta, 
and Santi [2]. A closed-form expression for the timestationary density of a node position is obtained only for random-waypoint on a one-dimensional interval; for two dimensions only approximations are obtained. Note that in Theorem 5 we do have an exact representation of the distribution of mobile location as a marginal of a distribution with a known density. Neither [8] nor [2] consider how to run perfect simulations. It is the original finding of Yoon, Liu, and Noble [15] that the default setting of the classical random-waypoint is in fact ill-defined. The default random-waypoint assumes the event-stationary distribution of the speed to be uniform on an interval $(0, v \max ]$. The authors found that if a node is initialized such that origin is a waypoint, the expected speed decreases with time to 0 . This in fact corresponds to an infinite event-average time between two waypoints, which as we show in Section IV, corresponds to the absence of stationary regime. In a subsequent work [16], the same authors advocate to run sound mobility models by initializing a simulation by drawing a sample of the speed according to its time-stationary distribution. We remark that speed is only a partial state of a node; in this paper, we look at the complete state of the node mobility. For the last reason, the authors in [16] do not completely solve the problem of running perfect simulations. Another related work is that of Lin, Noubir, and Rajaraman [12] that studies a class of mobility models where travel distance and travel speed between transition points can be modeled as a renewal process. The renewal assumption was also made in [15], [16]. We note that this assumption is not verified with mobility models such as classical random-waypoint on any non-isotropic domain, such as rectangle, for example. The renewal assumption has been made largely to make use of a "cycle" formula from renewal theory. An elementary knowledge of Palm calculus tells us that "cycle" formula is in fact Palm inversion formula, which we used extensively throughout the paper, and that applies more generally; this renders the renewal assumption unnecessary. Perhaps the work closest to ours is that of Navidi, Camp, and Bauer in [14], [13]. As discussed in Section I-C, we provide a systematic framework that allows to formally prove some of the implicit statements in [13] and generalize to a broader class. Further, our perfect sampling algorithm differs in that it works even when geometric constants are not a priori known.

\section{CONCLUSion}

Our perfect sampling algorithm is implemented to use with ns-2 to produce perfect simulations for a broad set of random trip mobility models. The code is freely available to download from:

\section{http://icalwww.epfl.ch/RandomTrip}

This web page contains also further pointers to random trip mobility models.

\section{ACKNOWLEDGMENTS}

The perfect sampling ns-2 code pointed out in this paper was implemented by Santashil PalChaudhuri. M. V. is grateful to Srdjan Čapkun for referring him to the mobility problem studied in this paper. He also thanks Don Towsley for encouragement and discussions.

\section{REFERENCES}

[1] François Baccelli and Pierre Brémaud. Palm Probabilities and Stationary Queues. Springer LNS, 1987.

[2] Christian Bettstetter, Giovanni Resta, and Paolo Santi. The Node Distribution of the Random Waypoint Mobility Model for Wireless Ad Hoc Networks. IEEE Trans. On Mobile Computing, 2(3), July-September 2003.

[3] Ljubica Blažević, Jean-Yves Le Boudec, and Silvia Giordano. A Location Based Routing Method for Mobile Ad Hoc Networks. IEEE Trans. on Mobile Computing, 3(4), Dec 2004.

[4] Jean-Yves Le Boudec and Milan Vojnović. Perfect Simulation and Stationarity of a Class of Mobility Models. Technical Report EPFL/IC/2004/59, EPFL, July 2004. http://lcawww.epfl.ch/Publications/LeBoudec/lebvoj04.pdf.

[5] Josh Broch, David A. Maltz, David B. Johnson, Yih-Chun Hu, and Jorjeta Jetcheva. A Performance Comparison of Multihop Wireless Ad Hoc Network Routing Protocols. In Mobile Computing and Networking, pages 85-97, 1998.

[6] T Camp, J. Boleng, and V. Davies. A Survey of Mobility Models for Ad Hoc Network Research. WCMC: Special issue on Mobile Ad Hoc Networking: Research, Trends and Applications, 2(5):483-502, 2002.

[7] Erhan Çinlar. Introduction to Stochastic Processes. Prentice Hall, 1975.

[8] Christian Bettstetter, Hannes Hartenstein, and Xavier PerezCosta. Stochastic Properties of the Random Waypoint Mobility Model. ACM/Kluwer Wireless Networks, Special Issue on Modeling and Analysis of Mobile Networks 2003.

[9] Amit Jardosh, Elizabeth M. Belding-Royer, Kevin C. Almeroth, and Subhash Suri. Towards Realistic Mobility Models for Mobile Ad-Hoc Networking. In Proc. of ACM Mobicom 2003, pages 217-229, San Diego, CA, 2003.

[10] Jean-Yves Le Boudec. On the Stationary Distribution of Speed and Location of Random Waypoint. IEEE Trans. on Mobile Computing, accepted for publication.

[11] Jean-Yves Le Boudec. Understand the Simulation of Mobility Models with Palm Calculus. Technical Report EPFL/IC/2004/53, EPFL, June 2004.

[12] Guolong Lin, Guevara Noubir, and Rajmohan Rajamaran. Mobility Models for Ad-Hoc Network Simulation. In Proceedings of Infocom 2004, April 2004.

[13] William Navidi and Tracy Camp. Stationary Distributions for the Random Waypoint Model. IEEE Trans. on Mobile Computing, 3(1), 2004.

[14] T. Camp W. Navidi and N. Bauer. Improving the Accuracy of Random Waypoint Simulations through Steady-state Initialization. In Proceedings of the 15th Int'l Conference on Modeling and Simulation (MS '04), pages 319-326, March 2004.

[15] Jungkeun Yoon, Mingyan Liu, and Brian Noble. Random Waypoint Considered Harmful. In Proc of Infocom, 2003.

[16] Jungkeun Yoon, Mingyan Liu, and Brian Noble. Sound Mobility Models. In Proceedings of Mobicom, 2003. 